\title{
Revealing the Internal Structure and Local Chemistry of Nanocrystals Grown in Hydrogel with Cryo-FIB Lift-Out and Cryo-STEM
}

\author{
Michael J. Zachman ${ }^{1,2}$, Emily Asenath-Smith ${ }^{3}$, Lara A. Estroff ${ }^{2,3}$ and Lena F. Kourkoutis ${ }^{1,2}$ \\ 1. School of Applied and Engineering Physics, Cornell University, Ithaca, NY 14853, USA. \\ 2. Kavli Institute for Nanoscale Science, Cornell University, Ithaca, NY 14853, USA. \\ 3. Department of Materials Science and Engineering, Cornell University, Ithaca, NY 14853, USA.
}

Hydrogels, three-dimensional polymeric networks with entrapped solvents, have gained increasing interest in a number of fields, including novel crystal synthesis. Compared to solution-based processes, crystal growth in hydrogels opens new routes to controlling morphology and function. Additionally, hydrogels have found applications in biomedical and biological research due to their biomimetic properties, which allow them to imitate the conditions surrounding cells. Understanding processes in hydrogels requires gaining access to their internal structures. Commonly, this requires removal of the liquid from the sample, as the hydrogel will dehydrate upon entering the vacuum of the microscope. Artifacts due to drying, however, can prevent imaging of the samples' native structure.

Cryo-transmission electron microscopy (TEM) is a well-established technique in biology for imaging the near-native structure of thin hydrated specimens [1]. Thicker materials, however, require sample thinning in order to access the hidden structures and interfaces within. Over the last decade, cryofocused ion beam (FIB) milling approaches were developed for on-grid thinning of frozen samples up to microns thick [2]. For larger samples, an alternative approach, cryo-FIB lift-out, has recently been demonstrated [3-5]. In this method a thin slice, or lamella, is removed from the frozen bulk sample, attached to a TEM grid and thinned to electron transparency, while avoiding de-vitrification during the entire process. Here, we report progress in this technique, showing the ability to produce high-quality TEM lamellas from a hydrogel sample. Using cryo-scanning transmission electron microscopy (STEM) we image the structure of nanocrystals grown inside the hydrogel, as well as track local changes in chemistry and bonding with electron energy loss spectroscopy (EELS).

For this work, iron chloride hexahydrate solution was added to the surface of a silica hydrogel and allowed to diffuse throughout its interior. Subsequent heating resulted in the growth of nanoparticles of an iron (oxy-, hydr-)oxide phase within the hydrogel. These structures were stabilized by snap-freezing in supercooled nitrogen, and then loaded into the cryo-FIB equipped with a Quorum PP3010T cryosystem. Figure 1 shows steps in the cryo-FIB lift-out process, from bulk sample to final lamella. To avoid instabilities due to sample charging, we first sputtered a thin layer of platinum on the surface of the hydrogel, which allowed preparation of a lamella with uniform thickness $(2-5 \mu \mathrm{m}$ thick, before liftout). Final thinning to $150-180 \mathrm{~nm}$ was performed with the lamella attached to the TEM grid (Fig. $1 \mathrm{c}, \mathrm{d})$.

The structure of the lamellas and the hydrogel-embedded nanoparticles were examined down to the nanoscale using annular dark field cryo-STEM. Convergent beam electron diffraction confirmed the particles' crystallinity. In addition, a comparison of the local bonding environments of the hydrogel and nanoparticles was obtained using EELS (Fig. 2). The potential impact of cryo-FIB lift-out in combination with cryo-STEM/EELS goes well beyond the field of crystal synthesis. In batteries and fuel cells for example, many critical liquid-solid interfaces are present, which have not been analyzed at high spatial resolution with the liquids intact. 
[1] J. Dubochet et al., Q. Rev. Biophys. 212 (1988), p. 129-228.

[2] M. Marko et al., J. Microsc. 2221 (2006), p. 42-47.

[3] S. Rubino et al., J. Struct. Biol. 180 (2012), p. 572-576.

[4] N. Antoniou, et al., 38th International Symposium for Testing and Failure Analysis (2012), p. 399.

[5] C. Parmenter, et al., Microsc. Microanal. 20 (Suppl 3) (2014), p. 1224.

[6] This work made use of the Cornell Center for Materials Research Shared Facilities which are supported through the NSF MRSEC program (DMR-1120296).
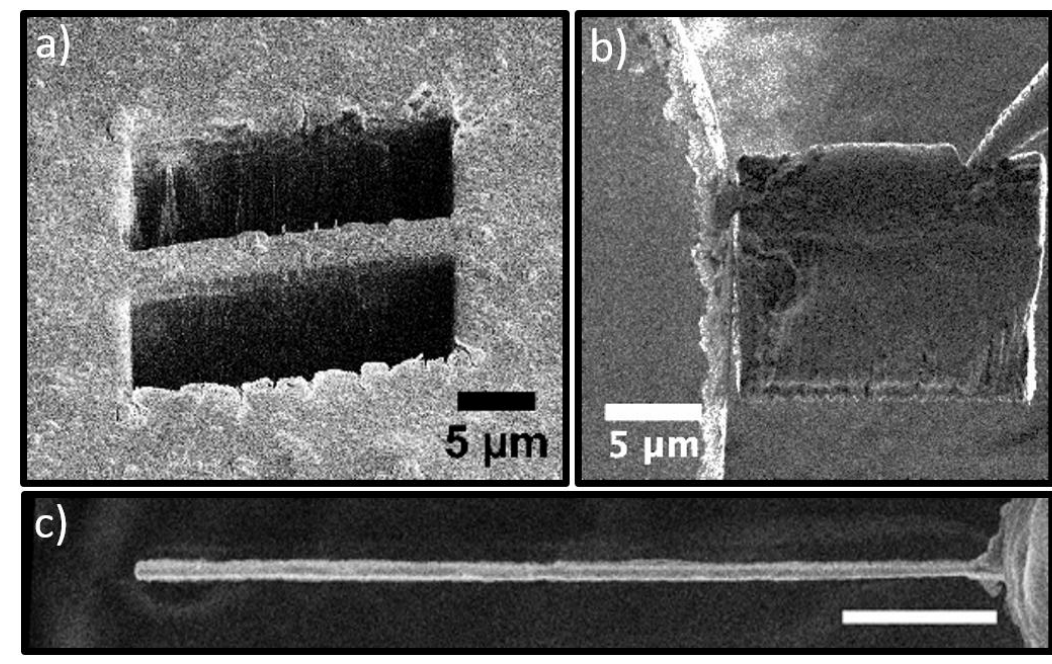

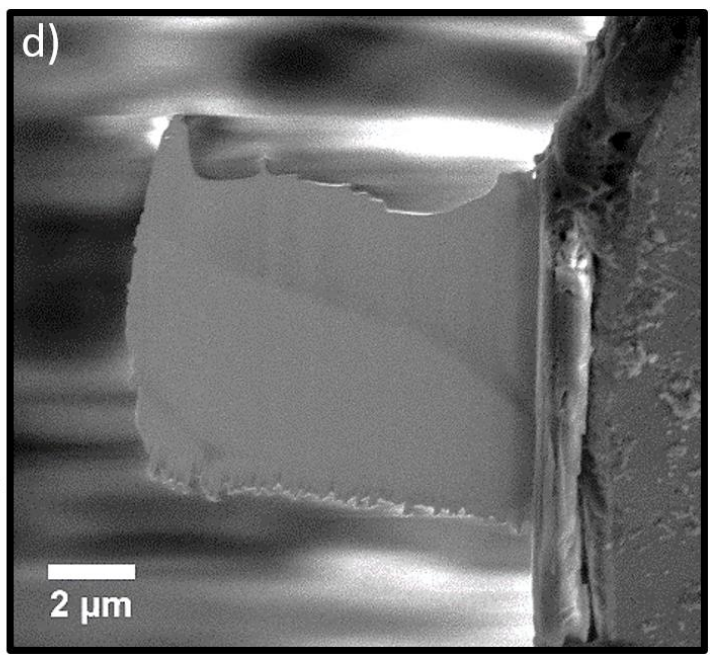

Figure 1. Preparation of electron transparent lamellas of frozen-hydrated hydrogel specimens by cryoFIB lift-out: (a) Site-specific milling of a lamella from the bulk hydrogel sample. A sample temperature below the vitrification point is maintained using a gas-cooled Quorum cryo-stage. (b) Attachment of the frozen lamella to a TEM grid, using a cryogenically cooled OmniProbe needle and GIS water vapor. (c) Thinning to electron transparency (150-180 $\mathrm{nm}$ here, $2 \mu \mathrm{m}$ scale bar). (d) A completed lamella imaged in the cryo-FIB and ready for transfer into the cryo-STEM.
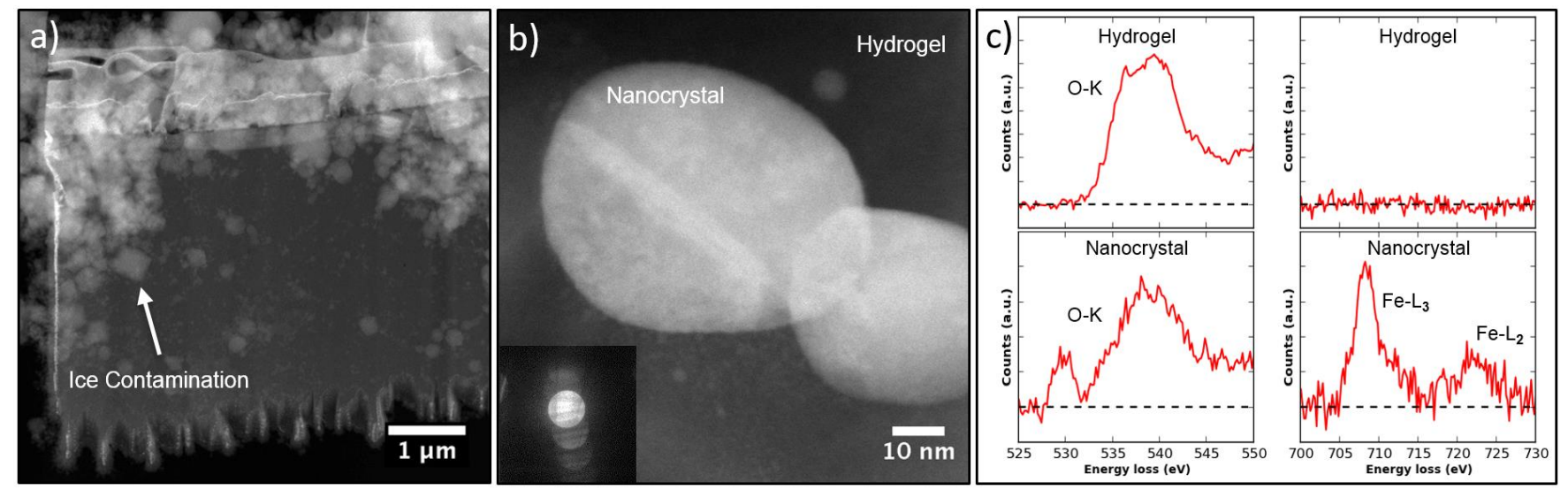

Figure 2. Annular dark field (ADF) cryo-STEM imaging of frozen-hydrated hydrogel lamellas prepared by cryo-FIB lift-out. Transfer from the cryo-FIB to the cryo-STEM can result in ice contamination (a), which hampers structural and spectroscopic analysis. Cryo-ADF-STEM of a contamination-free lamella reveals the structure of embedded iron (oxy-, hydr-)oxide phase nanoparticles (b). Crystallinity of these nanoparticles is confirmed by convergent beam electron diffraction ( $b$, inset), and information about local bonding can be extracted using electron energy loss spectroscopy (c). 